\title{
Les forums virtuels : ressource pour le développement des pratiques et du bien-être des professionnels
}

Virtual forums : a resource for professional development of practices

and wellbeing

\section{Magali Prost, Béatrice Cahour et Françoise Détienne}

\section{OpenEdition}

Journals

Édition électronique

URL : http://journals.openedition.org/activites/2827

DOI : $10.4000 /$ activites.2827

ISSN : $1765-2723$

Éditeur

ARPACT - Association Recherches et Pratiques sur les ACTivités

Référence électronique

Magali Prost, Béatrice Cahour et Françoise Détienne, «Les forums virtuels : ressource pour

le développement des pratiques et du bien-être des professionnels », Activités [En ligne], 13-2 | 2016,

mis en ligne le 15 octobre 2016, consulté le 21 avril 2019. URL : http://journals.openedition.org/ activites/2827 ; DOI : 10.4000/activites.2827

Ce document a été généré automatiquement le 21 avril 2019

\section{(c) $(1) \Theta$}

Activités est mis à disposition selon les termes de la licence Creative Commons Attribution - Pas d'Utilisation Commerciale - Pas de Modification 4.0 International. 


\title{
Les forums virtuels : ressource pour le développement des pratiques et du bien-être des professionnels
}

\author{
Virtual forums : a resource for professional development of practices
}

and wellbeing

Magali Prost, Béatrice Cahour et Françoise Détienne

\section{NOTE DE L'ÉDITEUR}

Article reçu le 03/02/2016, accepté le 30/06/2016

Les évolutions technologiques offrent aux professionnels l'opportunité de construire de nouveaux espaces pour parler de leur travail et de leurs difficultés. Ils interagissent en mobilisant des technologies tels que des forums, et ces interactions soutiennent le développement de communauté en ligne (Preece, Maloney-Krichmar, \& Abras, 2003). Les professionnels investissent de plus en plus ce type d'espaces virtuels qui se trouvent en dehors de leur institution, en vue d'échanger sur leurs pratiques et leurs éventuelles difficultés rencontrées au travail. Cet article s'intéresse au développement du pouvoir d'agir des professionnels par l'intermédiaire de ces nouveaux instruments (Rabardel, 1985) que sont les forums entre pairs partageant un même métier. Le forum dont il est question est structuré par les membres eux-mêmes, en dehors de l'organisation pour laquelle ils travaillent. Le forum est ainsi un soutien à leur activité professionnelle. Nous nous intéressons au rôle des échanges entre participants dans le développement de la pratique professionnelle et du bien-être (et plus largement de la santé), avec un intérêt pour le réinvestissement dans le réel des connaissances échangées dans la sphère virtuelle. Cette visée transformative des échanges, avec une focale sur les apports et limites des forums pour les professionnels dans leur contexte d'usage, n'est pas sans lien avec le cadre de la clinique de l'usage (Bobillier Chaumon, 2016) et s'inscrit particulièrement dans celui de l'ergonomie constructive (Falzon, 2013). 
2 Nous commencerons par présenter un bref état de l'art sur le développement du pouvoir d'agir, sur les communautés de pratiques virtuelles et leurs liens avec le développement des pratiques professionnelles, et la place du versant émotionnel dans les communautés virtuelles. Nous détaillerons ensuite la méthodologie utilisée dans cette étude, avec des méthodes multiples tels qu'un questionnaire, une analyse de fils de discussions et des entretiens d'autoconfrontation. Puis, nous présenterons les résultats et en proposerons une discussion organisée en différents points en lien avec la littérature.

\section{1. État de l'art}

\subsection{Clinique de l'usage et développement du pouvoir d'agir}

3 La clinique de l'usage, dont il est question dans cet article, est une approche écologique de l'acceptation située des technologies. Celle-ci est définie comme la "mise à l'épreuve de la technologie dans son contexte d'usage [permettant] d'évaluer concrètement ses apports et ses limites, et de définir ainsi son intérêt par rapport à l'activité et aux projets de l'individu» (Bobillier Chaumon, 2016, p. 6). L'étayage théorique de cette approche s'appuie d'une part sur l'appropriation technologique (Rabardel, 1985), et d'autre part sur les modèles de l'activité (Vygotski, 1934/1997).

4 La genèse instrumentale (Rabardel, 1985) met en évidence que l'artefact (ici technologique) devient un instrument par la construction de l'individu. L'instrument est façonné par les individus, de par leurs actions et leurs interactions (instrumentalisation), et dans un mouvement inverse, les individus sont transformés par les objets technologiques (instrumentation). Selon Bobillier Chaumon (2016), ce processus d'appropriation est une condition initiale à l'acceptation technologique.

5 Les approches de l'activité, initiées par Vygotski (1934/1997), sont au fondement de différents courants théoriques telles que les théories de l'activité (Engestrom, 1987), la clinique de l'activité (Clot, 2004) ou encore l'ergonomie constructive (Falzon, 2013). Ces différentes approches ont en commun de mettre l'accent sur le caractère incertain, situé et émergent de toute activité, considérée comme source de développement. Lorsque l'activité est médiée par la technologie, l'expérience vécue, les usages effectifs et leurs effets sur les pratiques deviennent au centre des préoccupations pour comprendre le processus d'acceptation (Bobillier, 2016).

6 Les différentes approches théoriques précédemment mentionnées partagent une même finalité, celle de développer le pouvoir d'agir des individus. Ce concept connaît quelques nuances selon l'approche qui le mobilise. Aussi, l'approche instrumentale (Rabardel, 1985), qui s'intéresse au «sujet capable», fait une distinction entre pouvoir d'agir et capacité d'agir. L'individu dispose de ressources constitutives de ses capacités d'agir - ce que l'individu est capable de faire dans ses différents domaines d'activité - et de son pouvoir d'agir - ce qu'il lui est possible effectivement de faire compte tenu des caractéristiques des situations, soit l'actualisation des capacités d'agir dans une situation singulière (Gouedard \& Rabardel, 2012 ; Rabardel, 2005).

7 L'approche de la clinique de l'activité (Clot, 2008 ; Clot \& Simonet, 2015) n'opère pas cette distinction potentiel/actualisé (capacité d'agir/pouvoir d'agir) et envisage le pouvoir d'agir dans une relation dyadique entre individu et situation. Le développement du pouvoir d'agir est ainsi considéré comme la «réalisation effective de l'élargissement du champ des 
actions (...), le développement du "rayon d'action" de l'activité des sujets dans leur milieu professionnel » (Clot \& Simonet, 2015, p. 38). Le développement de ce pouvoir d'agir transite par le renouvellement du sens et de l'efficience de l'activité en situation.

8 Dans un même ordre d'idée, l'approche de l'ergonomie constructive (Falzon, 2013) cherche à promouvoir des environnements capacitants, environnements qui favorisent le développement du pouvoir d'agir (Arnoud \& Falzon, 2013). La manière d'envisager ici le pouvoir d'agir est proche de celle de l'approche instrumentale. L'objectif est de chercher à développer conjointement les individus, les collectifs et les organisations (Barcellini, 2015 ; Mollo \& Nascimento, 2013) afin que ces dernières « contribuent simultanément et de manière pérenne à l'amélioration du bien-être des salariés, au développement des compétences et à l'amélioration de la performance. » (Mollo \& Nascimento, 2013, p. 2).

Les travaux de Fernagu-Oudet (2012, 2014, 2016 ; Prost \& Fernagu-Oudet, 2016), héritage des travaux de Sen $(2001,2003)$, s'inscrivent dans cette lignée des travaux sur les environnements capacitants tout en rappelant les travaux de Rabardel, et s'intéressent à la conversion des capacités d'agir (également appelées capabilités - entendues comme la possibilité effective qu'un individu a de choisir diverses combinaisons de fonctionnements) en pouvoir d'agir (capacités d'action et de choix) au travers de l'identification de facteurs de conversion. Ces derniers sont un ensemble de facteurs - individuels, sociaux, organisationnels - qui facilitent (facteur de conversion positif) ou entravent (handicap de conversion) la capacité d'un individu à faire usage des ressources pour les «convertir» en réalisations concrètes (Bonvin \& Farvaque, 2007; FernaguOudet, 2012). En l'absence de facteurs de conversion adéquats, les ressources ne peuvent pas se traduire en pouvoir d'agir, l'actualisation ne se fait pas. Cette approche de l'ergonomie constructive mise en perspective dans le cadre des environnements capacitants nous semble particulièrement intéressante à adopter dans la mesure où elle permet de faire le lien entre celles présentées plus haut, et y ajoute la question de la liberté de choix des individus. L'environnement capacitant, selon cette logique, est un environnement qui donne les moyens à l'individu de développer son autonomie de choix à agir de telle ou telle manière. Cela revient à favoriser le développement de marges de manœuvre situationnelles ${ }^{1}$ des individus (Coutarel, Caroly, Vézina, \& Daniellou, 2015) en développant «durablement les possibilités (collectives, institutionnelles, temporelles...) de débats de normes, d'explicitation des valeurs qui les sous-tendent et d'arbitrages par ceux que ces débats traversent et affectent. » (ibid., p. 18). Les débats collectifs favorisent l'instauration de règles (e.g. Caroly, 2011) et ouvrent l'espace des possibles.

10 Les travaux sur les environnements capacitants s'intéressent encore peu à la sphère virtuelle, à l'exception du travail de Barcellini (2015) qui a montré que les espaces de conception en ligne "open source" peuvent être considérés comme des espaces capacitants favorisant le processus d'élaboration de règles et d'explicitation des critères de qualité du travail.

11 Dans cet article, nous envisageons le pouvoir d'agir sous l'angle du développement des pratiques et du bien-être des individus. Il nous semble important de prendre en compte simultanément ces deux aspects pour que le pouvoir d'agir soit appréhendé plus largement qu'au travers des aspects socio-cognitifs de la pratique professionnelle, et que la manière dont les individus vivent émotionnellement ce développement soit prise en compte. Autrement dit, il s'agit, au-delà du pouvoir d'agir, de considérer le pouvoir d'agir sereinement. 

permet le déploiement du pouvoir d'agir des enseignants du primaire, observé par le développement des pratiques et du bien-être des professionnels concernés. Également, nous cherchons à identifier l'existence de facteurs liés du forum qui participent à l'adhésion des participants (facteurs de conversion). La partie suivante montre que dans le cadre de communautés de pratique virtuelles, les forums peuvent être un soutien au développement professionnel.

\subsection{Développement professionnel et communauté de pratique virtuelle}

13 Le phénomène, observé depuis quelques années, de regroupements de professionnels dans la sphère virtuelle fait écho à la littérature sur les communautés de pratique (Lave \& Wenger, 1991; Wenger, 1998) notamment virtuelles (COPv). Ces communautés virtuelles ont comme caractéristiques de regrouper des personnes dispersées géographiquement, partageant une pratique et des préoccupations, avec une organisation informelle (qui peut évoluer vers une organisation plus formelle) pour développer des connaissances, améliorer leurs savoirs et leurs savoir-faire à travers des interactions régulières et soutenues (MaKinster, Barab, Harwood, \& Andersen, 2006; Maloney-Krichmar \& Preece, 2005 ; Wenger, 1998). Chaque membre alimente la communauté grâce à son expérience et s'appuie en retour sur les connaissances des autres pour améliorer sa pratique. Les communautés de pratique sont considérées comme un «noyau» de compétences (Charlier \& Henri, 2004).

14 Un parallèle peut être fait entre les activités réalisées dans les COPv, tels que la confrontation de points de vue, le partage d'expérience, l'élaboration de savoirs partagés, etc. (Wenger, 1998) et celles qui se produisent dans les séances d'analyse réflexive collective décrites par Mollo et Nascimento (2013) dans la perspective de l'ergonomie constructive. Les compétences se construisent en partie par une activité réflexive dans laquelle une expérience située est l'objet d'un travail d'élaboration par le sujet (Weill-Fessina \& Pastré, 2004). La pratique réflexive désigne une « forme de réflexion sur l'action, menée en dehors du cadre fonctionnel immédiat, et permettant l'analyse critique individuelle ou collective d'une situation de travail singulière ou d'une famille de situations.» (Mollo \& Nascimento, 2013, p. 2). Elle est le moteur de l'activité constructive orientée vers le développement, la transformation de la personne (contrairement à la dimension productive qui vise la réalisation de tâches - Samurçay \& Rabardel, 2004). Certaines conditions sont nécessaires pour que la pratique réflexive puisse se faire (Mollo \& Nascimento, 2013), notamment un focus sur une activité réelle de travail (comprenant les récits de situations mettant en exergue les conditions réelles de réalisation de travail) ; la rencontre régulière d'un collectif pérenne; l'évaluation et l'élaboration collective des solutions et les différentes façons de réaliser la tâche ; et l'implication de l'organisation (lorsque ces pratiques réflexives sont institutionnalisées).

15 L'individu, à travers cette activité d'analyse, construit des connaissances sur la manière de traiter un problème et sur sa capacité à évaluer ses ressources pour les adapter à la situation (Weill-Fassina \& Pastré, 2004). Réalisée de manière collective, l'activité réflexive permet de faire évoluer les représentations sur une situation (ou une solution), de prendre conscience de l'existence d'autres manières de réaliser l'activité, de faire une analyse de ses propres savoirs et savoirs-faire sur la base de ceux des autres, d'élaborer de 
nouveaux savoirs (Mollo \& Nascimento, 2013, p.4). Par conséquent, cela favorise le développement des capacités des individus à faire face à la variabilité des situations ainsi que des marges de manœuvre en augmentant le nombre de solutions alternatives possibles (ibid.).

La mise en récit des expériences participe à la construction d'une mémoire de groupe à des fins "d'apprentissage, de capitalisation des connaissances ou de soutien aux activités fonctionnelles de la communauté» (Beaujouan, Coutarel, \& Daniellou, 2013, p. 31; Soulier, 2006). Cette mise en récit est donc un vecteur important pour construire du sens et des pratiques professionnelles, que les théories de l'apprentissage expérientiel ont bien mis en évidence (Bourassa, Serre, \& Ross, 1999).

L'objectif de notre étude est notamment de savoir si le forum étudié est un espace qui soutient le développement de la pratique professionnelle et si les connaissances acquises au travers des échanges entre pairs sont remobilisées en situation.

\subsection{Bien être, partage d'émotions et communautés virtuelles}

Si le lien avec la part épistémique du travail est ici assez clair, il convient de ne pas omettre la partie émotionnelle, liée à la question du bien-être des individus. L'approche ergonomique qui s'intéresse au bien-être des travailleurs met en lien le travail et la santé psychique (Vézina, 2002). La souffrance psychique est favorisée par la diminution, voire la destruction de la capacité d'agir, du pouvoir-faire, ressentie comme atteinte à l'intégrité de soi (Clot, 2004). Apparaissent alors des risques psychosociaux, faisant référence au vécu subjectif de la situation et à l'évaluation des capacités à faire face d'un individu (Lancry, Grosjean, \& Parmentier, 2008, cités par Marc, Grosjean, \& Marsella, 2011). L'individu peut trouver une issue à cette souffrance dans le collectif. Mais, lorsque ce dernier est défaillant, la souffrance peut devenir pathogène, si l'individu n'a pas de ressources personnelles qui lui permettent d'installer une distance protectrice (ou créatrice) vis-à-vis de son travail. La possibilité de débattre sur les contraintes et les ressources du travail favorise le développement des individus, des collectifs, de l'organisation, qui se répercute sur le bien-être des individus (Caroly \& Barcellini, 2013 ; Mollo \& Nascimento, 2013).

19 L'isolement professionnel, facteur de risques psycho-sociaux, est un processus pouvant s'inscrire sur un continuum dont les extrêmes sont le sentiment d'isolement et le sentiment de solitude (Marc et al., 2011). Ces auteurs insistent sur le besoin de partage et d'échanges, à la fois pour construire un référentiel commun et pour l'entraide émotionnelle. Cela fait écho aux travaux de Rimé (2005) qui montrent que le besoin de partage social ${ }^{2}$ des émotions est une conséquence caractéristique de l'expérience émotionnelle et qu'il intervient dans le processus de régulation des émotions. Ce phénomène permet, par le récit de son expérience, de donner du sens aux situations, de s'en distancier par l'élaboration, et lorsque la réponse de l'auditeur est adaptée, le partage permet de «stimuler la conviction d'être capable de maîtriser les situations " (ibid, p. 194).

20 Les forums d'échanges pourraient ainsi être un lieu de (re)constitution d'un collectif, différent de celui côtoyé quotidiennement, et donner la possibilité d'échanger sur ce qui pose problème dans la pratique professionnelle, ce qui n'est pas compris, ce qui impossible à faire, ce qui est difficile voir douloureux de faire, etc. 
Comme nous l'avons vu dans la partie précédente, les communautés virtuelles (soutenues par des forums de discussion) peuvent être des lieux de production de connaissances, mais elles peuvent aussi être des lieux de partage d'émotions (Derks, Fischer, \& Bos, 2007). L'étude plus globale dans laquelle se situent les analyses exposées dans cet article a montré que le forum d'enseignants dont il est question ici est une communauté hybride, teintée à la fois d'échanges épistémiques et d'échanges de nature émotionnelle (Prost, 2012 ; Prost, Cahour, \& Détienne, 2013, 2014). Aussi il existe, en parallèle des travaux sur les communautés de pratique (axés sur l'amélioration de la pratique professionnelle et la production de connaissances), des travaux qui s'intéressent à cette dimension émotionnelle dans les communautés virtuelles. Ces études se centrent sur des communautés de soutien (principalement des patients ou des personnes âgées) et ne s'intéressent pas (ou peu) aux communautés de professionnels. Elles postulent que le dévoilement de soi ("self-disclosure ») serait plus important et plus spontané que dans les relations non médiatisées (Lewkowicz, Marcoccia, Atifi, Bénel, Gaglio, Gauducheau et al., 2008; Park \& Floyd, 1996; Walther \& Boyd, 2002 ; Wright \& Bell, 2003), y compris dans les situations non propices telle que la résolution de problème (Joinson, 2001). L'anonymat serait un élément fondamental facilitant cette expression émotionnelle, notamment négative, en raison du caractère moins menaçant de leur exposition (Caplan, 2003 ; Caplan \& Turner, 2007 ; Walther \& Boyd, 2002). Différentes fonctions sont associées au dévoilement émotionnel (Tichon \& Shapiro, 2003) telles que : demander du soutien de manière implicite (Pfeil \& Zaphiris, 2007 ; Preece, 1999 ; Prost et al., 2014); apporter du soutien en montrant qu'une solution est envisageable; partager des moments de complicité avec un autre participant; permettre aux participants de procéder à une comparaison sociale qui le plus souvent est source de réassurance (Helgeson \& Gottlied, 2000 ; Walther \& Boyd, 2002).

Les participants de ces communautés témoignent de leur besoin de similarité. Savoir que d'autres personnes vivent la même situation qu'eux les aide à réduire leur sentiment d'isolement (eg. Malik \& Coulson, 2008; Walther et Boyd, 2002). Ce phénomène correspond à la recherche de comparaison sociale, caractéristique des personnes se trouvant dans une situation émotionnellement difficile (Wills, 1981). Le partage d'expérience similaire, notamment les situations stressantes, permet de valider, normaliser l'expérience et réduire l'isolement émotionnel (Helgeson \& Gottlied, 2000).

La communication « empathique » dans les communautés en ligne est traduite par de la compréhension, du dévoilement de soi émotionnel, le témoignage d'un vécu similaire et de l'intérêt (Pfeil \& Zaphiris, 2007). Les personnes qui partagent des situations similaires sont plus susceptibles d'avoir une discussion avec un fort contenu empathique ( ibid.), ce qui conduirait certains participants à établir des relations plus intimes qu'en face à face (Hian, Chuan \& Trevor, 2004; Pfeil, Zaphiris \& Wilson, 2009; Tidwell \& Walther, 2002), les relations pouvant évoluer, avec le temps, vers d'autres moyens de communication (e.g. Parks \& Floyd, 1996). La participation aux communautés virtuelles aurait une influence sur l'amélioration du bien-être psychologique des participants (Braithwaite, Waldron, \& Finn, 1999; Merseth, 1992 ; Pfeil et al., 2009 ; Wright, 2002 ; Wright \& Bell, 2003).

Un objectif de l'étude présentée ici est de savoir si le forum analysé est un espace qui permet aux participants de partager des situations de travail émotionnelles difficiles, et d'atténuer ce vécu. 


\section{Synthèse des questions de recherche} une rapide présentation du forum de discussion étudié et du métier d'enseignants du primaire.

\subsection{Présentation du forum} enseignants du primaire (http://forums-enseignants-du-primaire.com, EDP). Conçu en 1998 par un jeune enseignant en manque de formation, de contacts et d'informations (propos recueillis auprès du webmaster lui-même), il est le plus important des sites dédiés aux professeurs des écoles. La charte du site définit le forum comme "un espace de discussion, d'échanges et d'entraide». De nombreux professionnels (180 000 inscrits en décembre 2015) s'y engagent pour parler de leur travail, au sein de discussions classées par thématiques (e.g. " organiser, préparer et gérer une classe en élémentaire »). Les différents forums sont très actifs en termes de nombre d'interactions, de nombreux messages alimentent chaque jour les discussions.

31

Le forum requiert d'être inscrit et identifié pour participer aux échanges, la possibilité est toutefois laissée aux internautes de lire le contenu sans être authentifiés. Chaque participant doit donc utiliser un pseudonyme lorsqu'il veut contribuer aux échanges, ce 
qui facilite la reconnaissance et le contact entre les membres. Des informations personnelles peuvent être divulguées, sans que cela ne soit obligatoire (région, âge, intérêts/loisirs, etc.). Le site propose de nombreuses fonctionnalités telles que les smileys, la citation électronique, l'insertion d'avatars et de signatures. La modération du forum est faite a posteriori par des modérateurs choisis par l'administrateur du site (le plus souvent les meilleurs contributeurs), autrement dit les messages sont publiés avant d'être vérifiés par l'équipe de modérateurs.

D'après le webmaster (interrogé pour l'étude), une très grande proportion de femmes compose la communauté (environ $1 \%$ de membres déclarent être des hommes), ce qui semble conforme à la répartition hommes/femmes dans ce métier ${ }^{3}$. La majorité des membres auraient entre 26 et 40 ans et viendrait toujours selon le webmaster «pour obtenir de l'aide dans la réalisation de leurs séances, trouver des pistes concrètes pour faire la classe et partager leurs expériences. Je crois que ce qui compte le plus, pour beaucoup, c'est le besoin de se rassurer ".

Une précédente étude (Prost, 2012) a mis en évidence que les thèmes discutés sur ce forum étaient principalement liés au contenu de la pratique professionnelle (contenu, méthode et outil pédagogiques). Les aspects relationnels avec les collègues et la hiérarchie sont quasiment absents des échanges, non pas qu'ils soient absents des problèmes rencontrés, mais les participants n'en font pas part (Prost et al., 2013). Les participants qui initient une discussion expriment des émotions (notamment doute, expression d'une difficulté, stress, déception, frustration, insatisfaction). Les demandes exprimées dans les messages concernent principalement du partage d'expérience, des demandes d'aide instrumentale, de conseils, d'informations et d'évaluations. Il n'y a pas, ou très peu, de demandes explicites de soutien émotionnel, mais cette attente est socialement masquée et implicitement manifestée via le dévoilement de soi émotionnel ( ibid.). L'amélioration de la pratique est le motif mentionné par quasiment l'ensemble des participants à l'étude (Prost, 2012), élément faisant référence pour eux par exemple à la découverte et l'utilisation de nouvelles méthodes pédagogiques, à une meilleure prise en compte de la diversité des élèves.

\subsection{Présentation du fonctionnement de l'éducation nationale et du métier d'enseignant du primaire}

Le Ministère de l'Éducation Nationale (MEN) est le responsable de l'élaboration et de la mise en œuvre des programmes scolaires. Ces derniers ont été proposés par le Conseil Supérieur des Programmes (CSP) après une lettre de mission du MEN qui en contrôle ensuite l'application. Il est assisté par l'Inspection générale de l'Éducation nationale (IGEN) et l'Inspection générale de l'administration de l'Éducation nationale et de la Recherche (IGAENR), qui ont vocation à inspecter et évaluer le fonctionnement du système éducatif. Au niveau des 30 académies, l'enseignement du premier degré est dirigé par des recteurs (nommés par le Ministre) qui organisent, contrôlent et évaluent les activités des professionnels par le biais des Directeurs Académiques des Services de l'Éducation Nationale (IA-DASEN) sur le plan départemental puis au niveau de circonscriptions, des Inspecteurs de l'Éducation Nationale (IEN). L'IEN est le supérieur hiérarchique direct des professeurs des écoles (PE). Il a pour mission de conseiller, d'inspecter, de noter les personnels des écoles maternelles et élémentaires de sa circonscription. Il inspecte régulièrement les $\mathrm{PE}$ et leur attribue une note qui peut 
participer à leur avancement de carrière. L'IEN est assisté par des conseillers pédagogiques dont la mission est d'accompagner pédagogiquement les $\mathrm{PE}$, la mise en œuvre de la politique éducative ainsi que de contribuer à leur formation initiale et continue. Les PE peuvent faire appel au conseiller pédagogique de leur département en cas de problèmes ou de difficultés, mais il est fréquent qu'ils émettent une certaine méfiance compte tenu de sa proximité avec l'IEN qui les évalue. Enfin, chaque établissement est dirigé par un directeur d'école qui n'exerce aucune fonction hiérarchique vis-à-vis des PE. Il veille au bon fonctionnement de l'école et au respect de la réglementation. Il est l'interlocuteur de la mairie, notamment pour les dotations et achats de matériel pédagogique ${ }^{4}$.

Le métier de professeur des écoles regroupe différentes tâches dont une partie, visible et facilement identifiée, est réalisée en classe lors de l'animation des séances, cinq jours par semaine auprès de jeunes enfants. L'autre partie, moins manifeste, concerne la préparation des séances et se réalise la plupart du temps au domicile des individus. Cette activité est souvent solitaire et les enseignants ont pour seule directive de leur hiérarchie de suivre le programme décidé au niveau du Ministère de l'Éducation. Ils ont toute latitude dans le choix des méthodes pédagogiques, de l'organisation en classe, etc. Les professeurs des écoles peuvent être confrontés à tous les niveaux de classe, allant de la maternelle $1^{\text {ère }}$ année au CM2. La répartition des classes est théoriquement faite de manière collégiale en fin d'année, avant les vacances d'été, et est ainsi susceptible d'être modifiée à chaque nouvelle rentrée de septembre. Hormis les établissements urbains qui accueillent de nombreux élèves, il n'existe souvent qu'une classe par niveau, ce qui entraine que les professeurs des écoles n'ont pas, la même année, d'homologues confrontés au même travail au sein de leur établissement.

\subsection{Méthodes}

\subsubsection{Stratégie de recherche}

Le pouvoir d'agir est appréhendé au travers d'une dimension constructive/développementale, liée au développement des pratiques professionnelles et leur réinvestissement en situation; et dans une dimension émotionnelle, liée au développement du bien-être des individus.

Ainsi, l'étude cherche à savoir si les échanges entre enseignants du primaire sur le forum permettent le développement des pratiques et le développement du bien-être des membres, et à quelles conditions (facteurs de conversion) les participants s'engagent sur le forum. Ces deux dimensions seront analysées à partir du discours des participants observé d'une part dans les échanges produits par les participants sur le forum (i.e. fils de discussion) et d'autre part, à travers les discours produits sur les apports de ces échanges pour la pratique professionnelle et le bien-être des membres. Pour ce faire, trois méthodes complémentaires de recueil de données ont été mises en place pour appréhender le développement des pratiques et du bien-être des participants: un questionnaire, des entretiens d'auto- 
confrontation basés sur les traces écrites des discussions et des fils de discussions sélectionnés sur le forum.

Nous avons ensuite réalisé, sur l'ensemble de ces données, une analyse de contenu orientée par les catégories proposées par Mollo et Nascimento (2013) concernant les effets bénéfiques de l'activité réflexive, à savoir :

- Le changement de point de vue. Cette première catégorie fait référence au renouvellement du sens, au décentrement ;

- La prise de conscience des autres formes de réalisation de l'activité ;

- L'analyse critique de ses savoirs et savoir-faire au regard de ceux d'autrui ;

- La construction de nouveaux savoirs et outils (élaboration individuelle et/ou collective).

Cette liste a été complétée par trois catégories faisant référence : (1) au réinvestissement des connaissances acquises sur le forum dans la pratique professionnelle ultérieure, (2) à la dimension émotionnelle, absente dans les catégories proposées par les auteures (témoignage de soutien émotionnel et amélioration de l'expérience émotionnelle), et (3) aux caractéristiques du forum qui conditionnent la participation des membres.

\subsubsection{Recueil de données}

Les trois méthodes utilisées dans cette étude (cf. Tableau 1) seront présentées dans cette partie.

\section{La diffusion d'un questionnaire en ligne}

41 Le questionnaire en ligne a été utilisé afin d'avoir accès à un nombre important de participants afin de recueillir des données riches, notamment sur les apports des échanges. Il était composé de questions ouvertes permettant d'interroger les participants sur leurs motivations à lire ou à participer à une discussion; sur les apports et satisfactions des échanges; sur le type de problèmes rencontrés ainsi que les comportements mis en place pour y faire face. Quelques questions fermées ont été formulées : outre les informations sociodémographiques (âge, genre, profession, région), les questions fermées étaient relatives à la fréquentation du forum, à un éventuel maintien du contact avec des participants et à la fréquence de rencontre de problèmes professionnels ${ }^{5}$.

42 Le questionnaire a été mis en ligne sur le forum de discussion (avec l'accord préalable du webmaster) pendant une période d'un mois. Les participants avaient ainsi la possibilité d'y répondre et de laisser ou non leurs coordonnées en vue d'un entretien pour affiner les données ainsi recueillies. Quatre-vingts réponses ont été obtenues et retenues pour analyse.

\section{La conduite d'entretiens d'auto-confrontation}

43 Neuf entretiens ont été réalisés avec des participants volontaires ayant préalablement répondu au questionnaire. L'objectif était d'étudier le vécu subjectif des interactions en ligne, ce qui n'y était pas exprimé et observable (voir Prost et al., 2013) et la manière dont ce qui s'échange sur les forums est réinvesti dans le réel du travail. confrontation avec comme les traces écrites des discussions recueillies sur le forum 
(faisant office de traces de l'activité des participants). Dans notre étude, l'entretien d'auto-confrontation est couplé à des relances d'entretien d'explicitation (Vermersch, 1994) afin de favoriser la mise en évocation du sujet (Cahour, 2006; Cahour, Salembier, \& Zouinar, 2016).

Les entretiens d'auto-confrontation se sont déroulés au domicile des participants, ont été filmés et intégralement retranscrits. La durée de chaque entretien a varié entre deux et trois heures.

\section{L'analyse de fils de discussion}

Nous avons réalisé une analyse de la nature des échanges (Bardin, 1977) au sein d'une centaine de discussions (pour plus de détails sur la méthode utilisée, voir Prost, 2012; Prost et al., 2014). Les critères de choix de ces discussions étaient les suivants :

1. les échanges devaient être centrés sur la pratique professionnelle, autrement dit ancrés dans le réel de situations spécifiques. Pour cela, nous avons effectué un premier tri des discussions selon qu'elles soient orientées vers l'échange d'informations factuelles, centrées sur des discussions autour du métier en général, ou axées sur la pratique concrète du métier. Seule cette dernière catégorie a été conservée ;

2. parmi ces discussions, nous avons sélectionné celles dans lesquelles les personnes ayant accepté un entretien ont participé (en vue de réaliser un entretien d'auto-confrontation). Au final, une vingtaine de fils a été analysée.

\subsubsection{Analyse des données}

Le tableau 1 synthétise et met en correspondance les questions de recherche, les méthodes utilisées et les traces recherchées dans les données recueillies. Un exemple est donné pour illustrer chaque critère :

Tableau 1 : Synthèse des questions de recherche, méthodes et analyses des traces. Table 1: Summary of research questions, methods and traces analyzes

\begin{tabular}{|l|l|l|l|}
\hline $\begin{array}{l}\text { Méthodes } \\
\text { Objectifs de } \\
\text { recherche }\end{array}$ & Questionnaire & $\begin{array}{l}\text { Entretiens d'auto- } \\
\text { confrontation }\end{array}$ & Fils de discussions \\
\hline
\end{tabular}




\begin{tabular}{|c|c|}
\hline $\begin{array}{l}\text { Développement } \\
\text { des pratiques }\end{array}$ & $\begin{array}{l}\text { *Changement de représentation / de point de vue } \\
\text { Exemple (questionnaire) : "les discussions apportent des éclaircissements, une } \\
\text { vision différente des choses. Lorsque nous avons une difficulté, les personnes qui sont } \\
\text { extérieures voient mieux que nous les solutions à envisager pour se sortir d'une } \\
\text { mauvaise passe" } \\
\text { *Prise de conscience des autres formes de réalisation de l'activité } \\
\text { Exemple (questionnaire): "Comme de nombreuses personnes fréquentent ces } \\
\text { forums, a fortiori des personnes qui ont une plus grande expérience que moi, je } \\
\text { profite de tous les conseils qu'elles peuvent me donner et de tout ce qui a pu être mis } \\
\text { en place dans leurs classes" } \\
\text { *Analyse critique de ses savoirs et savoirs-faire } \\
\text { Exemple (échanges écrits): "La 1ère année, j'avais bossé à fond la science et } \\
\text { l'histoire/géo, mais du coup, en anglais, c'était très très largement perfectible. En } \\
\text { français, j'étais 'au top' niveau productions d'écrits, littérature, mais pas au point en } \\
\text { lecture. La deuxième année, j'ai mis le paquet sur l'anglais, la lecture (avec des } \\
\text { résultats vraiment ++, de vrais progrès constatés chez les élèves), et du coup en } \\
\text { sciences, c'était franchement pas terrible. " } \\
\text { *Construction de nouveaux savoirs et outils } \\
\text { Exemple (entretien) : " on l'a construit ensemble ce livret, grâce à nos expériences, } \\
\text { chacune y a mis sa patte " } \\
\text { *Réinvestissement des connaissances acquises sur le forum dans sa } \\
\text { pratique professionnelle } \\
\text { Exemple (questionnaire) : "Je recherchais des exemples de progressions et } \\
\text { programmation (...), c'était bien agréable et rassurant de pouvoir en discuter sur le } \\
\text { forum etj'ai pu réutiliser ce qui a été transmis" }\end{array}$ \\
\hline $\begin{array}{l}\text { Développement } \\
\text { du bien-être }\end{array}$ & $\begin{array}{l}\text { * Amélioration / évolution positive de l'expérience émotionnelle } \\
\text { Exemple (entretien) : "Au niveau hyper concret, ça m'a vachement aidé. Et ça m'a } \\
\text { calmé aussi, car j'étais très énervée. C'est comme toujours, quand on a un truc sur le } \\
\text { cœur, ça fait du bien de le dire sinon ça aigrit. C'est toujours bien de pouvoir parler } \\
\text { quand on en a gros sur la patate, c'est toujours bien aussi de voir qu'il y a des gens } \\
\text { qui ne tombent pas dans le système" } \\
\text { * Témoignage de soutien émotionnel } \\
\text { Exemple (échanges écrits) : «Pour ce qui est de ne pas avoir le temps de tout } \\
\text { faire... celle ou celui qui te dirait être capable du contraire aurait un point commun } \\
\text { avec Pinocchio :D, Mais je comprends bien que cela peut stresser de se dire et ça je ne } \\
\text { l'ai pas fait et ça aussi... " (réassurance et compréhension) }\end{array}$ \\
\hline
\end{tabular}

L'ensemble des données recueillies (questionnaire, entretiens, discussions) a été analysé dans le but d'observer le développement des pratiques et du bien-être, ainsi que leurs conditions liées aux caractéristiques du forum (cf. Tableau 1).

Par ailleurs nous avons dégagé les facteurs de conversion liés au forum qui peuvent expliquer l'engagement des participants sur le forum. 


\section{Résultats}

50 l'expression émotionnelle liée aux situations de travail relatées, un besoin de décharge émotionnelle et des manifestations de soutien (réassurance, compréhension, encouragements, etc.). L'ensemble des fils de discussion contient une part plus ou moins d'émotions. Notons que l'intégralité des répondants au questionnaire mentionne l'existence (pour la plupart épisodiquement) de problèmes au travail qui pour deux tiers d'entre eux ont un retentissement sur leur bien-être psychologique et prennent la forme de nervosité, de découragement, de stress ou de préoccupations. Les principales sources des problèmes exprimés sont liées à la surcharge de travail et à la construction du contenu du travail. Le besoin de décharge émotionnelle et la recherche de soutien psychologique sont des attentes mentionnées dans plus de $70 \%$ des réponses au questionnaire et sont systématiquement retrouvées dans les entretiens d'autoconfrontation. Savoir que d'autres personnes vivent la même situation qu'eux aident les participants à réduire leur sentiment d'isolement, en témoigne cette participante lors d'un entretien "Quand je suis angoissée, je vais chercher des témoignages de gens qui vivent la même chose, et je vois que c'est pire que moi donc ça me rassure. C'est méchant, mais c'est vrai ». Les entretiens mettent en évidence que le soutien psychologique apporté par les échanges est favorisé par le fait de constater que d'autres vivent des situations similaires, d'être le destinataire de témoignage de soutien émotionnel (empathie, réassurance, etc.) et de trouver une solution à un problème :

"J'ai le sentiment d'être compris sur ce forum, nous sommes entre pairs et nos problématiques sont les mêmes. J'aime aussi pouvoir apporter mon aide aux collègues en galère; à là la différence des sphères familiales ou amicales qui écoutent, mais ne comprennent pas, sur ce forum j'ai l'impression de tout obtenir d'un coup : écoute, soutien, réconfort et solution » (questionnaire).

51 Les résultats de l'étude seront présentés selon quatre axes. Les trois premiers mettent en évidence l'apport développemental des échanges au niveau de l'élaboration collective de règles de métier, de l'élaboration collective d'outils et de savoirs et savoir-faire, notamment sur la gestion des relations. Le versant émotionnel sera illustré dans chacun d'eux. Le dernier axe est centré sur les facteurs de conversion liés au forum favorisant ainsi le développement du pouvoir d'agir. Nous montrerons pour chacun de ces thèmes comment le contenu des échanges permet le développement de la pratique et du bien-être à travers le réinvestissement en situation de travail.

\section{1. Élaboration de solutions et de normes}

Au sein des échanges, les participants discutent de leurs difficultés à interpréter ou à appliquer ce qui leur est demandé, ce qui est situation génératrice de surcharge de travail. Ils cherchent des solutions pour pallier une difficulté d'une ou plusieurs personnes en échangeant leur expérience et leur point de vue, et construisent ainsi de nouveaux savoirs et savoir-faire au regard de ceux dévoilés par les autres. En témoignent les propos en entretien d'une enseignante qui explique avoir remis en question la méthode pédagogique de lecture qu'elle utilisait au profit d'une autre décrite par une enseignante reconnue comme experte sur le forum: 
«Je suivais les aventures de la fameuse A, qui est une instit hors pair (...) je suivais beaucoup les discussions qu'elle avait avec les autres instits et je commençais à me dire au fil de l'année que ce n'était pas la bonne méthode que j'avais adoptée, que ce n'était pas ça qu'il aurait fallu faire, je faisais des correctifs aussi sur ma manière de faire. Toute l'année je travaillais sur "Rue des contes" et en même temps murissait l'idée de faire autrement l'année suivante et c'est ce que j'ai fait et je ne regrette pas » (entretien).

53 Cette enseignante, comme beaucoup d'autres, a procédé à une analyse critique de sa pratique grâce à la confrontation de celle des autres. Elle s'est s'approprier les savoirs et savoir-faire décrits par d'autres et les réinvestit dans sa pratique professionnelle. Cette participante a en outre mentionné l'amélioration de son expérience émotionnelle suite à l'intégration de cette nouvelle méthode dans sa pratique :

«ça a vraiment changé ma pratique professionnelle, il y a des choses dont j'étais un peu convaincue, mais je n'aurais pas eu le courage de les porter toute seule (...) le fait d'avoir des gens qui pensent pareil, c'est aussi un garde-fou, ça permet de se dire qu'on n'est pas dans la folie douce, ça m’a énormément, mais alors énormément rassurée » (entretien).

Une autre source d'échanges collectifs concerne les injonctions ministérielles relatives aux programmes qui apparaissent parfois compliquées à comprendre et à mettre en œuvre par les professionnels. L'extrait de fil de discussion suivant illustre ce point concernant les évaluations des élèves :

Initiateur «Je me pose des questions sur les livrets d'évaluation et cahiers de progrès pour la maternelle, car manifestement, je n'ai rien compris... Dans mon école, mes collègues ont choisi de faire un livret de compétences, et d'inscrire la date en face de cette compétence, une fois que celle-ci est validée. Pour la suite, nous utiliserons un cahier de progrès (dans lequel nous collerons des images ou des gommettes). A-t-on encore le droit d'utiliser un livret avec les compétences et un code (en lettres ou en chiffres) comme cela se faisait jusqu'à présent? »

Réactant 1 «tout le monde n'a pas compris la même chose à l'animation pédagogique sur le nouveau programme où tous les $\mathrm{PE}$ de mater de ma circo étaient conviés [smiley clin d'œil]. Dans notre école :

- nous avons rédigé "un cahier de progrès" qui recense en gros toutes les compétences éditées dans le nouveau programme (on a mis une partie "vivre ensemble" car ça nous tenait à cœur)

- dans ma classe et celle des TPS (et les autres vont sans doute suivre) on fait coller aux élèves dans leur classeur, les compétences quand elles sont acquises.

Comme dit Moustache il n'y a pas d'INTERDICTION formelle »

Réactant 2 «Je vais être assez terre à terre en vous posant 2 ou 3 questions qui me semble fondamentales: (...) Est-ce aux enseignants de devoir en plus de faire fonctionner leur classe, de fabriquer tout un tas de grilles de critères, de niveaux, de repères sachant que la progression des apprentissages est similaire d'un enfant à l'autre ? Ne serait-ce pas plutôt à nos chers hauts fonctionnaires de le faire au lieu de nous prendre pour des incapables en nous donnant des nouveaux programmes qui ne nous apprennent pas plus à enseigner qu'avant? Moi aussi j'ai créé des livrets d'évaluation pour m'aider à situer l'élève dans ses apprentissages, mais aucun n'a tenu plus de 2 ans. La raison première est l'impossibilité de bâtir une progression sérieuse à travers les années, chaque enseignant livré à sa représentation de l'apprentissage et au bien fondé de ce qu'il fait ne prend pas le temps du recul et de la discussion; le système actuel scolaire ne le permet pas ! (... )$»$

55 Cet exemple met en évidence les difficultés des enseignants à comprendre ce qui est attendu d'eux, le prescrit n'étant pas possible à appliquer dans le réel des contraintes de l'activité (temporelles ici), posant un problème pour concevoir l'outil imposé (ici le 
livret). Les échanges sur le forum viennent combler les lacunes de l'institution en termes de concertations et d'échanges. L'institution impose parfois des directives rigides, déconnectées de la réalité selon les participants. Les tentatives de l'institution d'imposer des critères de contrôle, dans l'esprit des méthodes de management de la qualité qu'on rencontre dans les entreprises, se heurtent ici à la réalité des pratiques. On voit que les professionnels tentent de s'y conformer, n'y arrivent pas, et en viennent progressivement à en mettre en doute le bien-fondé. On peut voir dans la mise en commun des critiques, les prémisses d'une résistance collective. On voit aussi comment certains discutent de la pertinence et remettent en question ce qui leur est demandé et évoquent les conditions dans lesquelles ces directives sont données, non propice à une mise en place satisfaisante de leur point de vue (réactant 2). L'ensemble de ces échanges participe à l'élaboration de solutions et de normes collectives.

De même, de nombreux participants considèrent leur formation initiale comme très insuffisante, estiment ne pas avoir les compétences pour concevoir des cours adaptés aux besoins des élèves, et vont chercher de l'aide sur le forum. L'extrait suivant illustre ce point :

« À l'IUFM [ancien ESPE], on ne nous apprend rien du tout. L'année dernière, sur le $\mathrm{CP}$, on n'a eu aucune info, qu'est-ce qu'on fait, par quoi on commence, on nous répond "vous vous ferez votre propre idée" (...) Ils ne veulent pas privilégier une méthode plus qu'une autre, ce qui fait qu'on ne sait pas si la syllabique marche mieux que la globale, par exemple. (...) Moi quand je suis arrivée, je n'ai pas eu le temps de choisir une méthode et tout était commandé pour la méthode Ribambelle et moi Ribambelle, je ne m'y fais pas du tout. (...) c'est ce que je suis venue chercher sur le forum, des gens qui utilisent d'autres méthodes et qui m'aident à trouver celle qui me convient » (entretien).

Un autre exemple, issu d'un entretien, illustre le rôle du forum comme soutien au changement de point de vue et à la prise de décision, ici ne pas réaliser une tâche considérée comme non adaptée (donnée par une collègue).

«L'année dernière, j'étais remplaçante et l'instit que je remplaçais m'avait demandé de finir le présent et de faire tout le futur en 3 semaines. Je m'étais dit "mais c'est pas possible" et j'étais allée voir sur le forum et on m'avait dit "mais c'est pas possible, elle se moque de toi, elle a rien foutu cette année et elle se rattrape sur toi". Du coup, je m'étais dit que tant pis, je ne ferais pas ce qu'elle m'avait demandé donc j'avais fini le présent et puis c'est tout. Moi toute seule, je ne l'aurais pas remis en cause, car elle était quand même expérimentée, moi ça m'a paru beaucoup, mais je me suis dit "je peux me tromper", mais non en fait, je ne me suis pas trompée, je l'ai vu grâce aux avis des gens sur le forum. »

Cet exemple montre le réinvestissement dans le réel du contenu des échanges, qui a conduit cette jeune enseignante à modifier ce qu'elle avait prévu de faire. Les retours de ses pairs l'ont rassurée dans l'évaluation qu'elle avait faite d'une prescription impossible à tenir, elle témoigne du bienfait émotionnel de ce fil de discussion "ça m'a redonné confiance et surtout le courage de ne pas essayer de tout faire ».

Une autre illustration de l'influence des échanges dans le changement de pratique est proposée dans l'extrait d'entretien suivant. La participante initie une discussion dans laquelle elle demande des techniques pour réaliser l'intégralité de son programme, ce qu'elle n'arrive pas à faire et ce qui génère chez elle un sentiment d'impuissance et d'angoisse. Les réponses qui lui sont faites sont un peu décalées par rapport à son attente initiale. Les réactants, souvent expérimentés, l'invitent plutôt à reconsidérer sa tâche et à sélectionner dans le programme les éléments pédagogiques les plus importants : 
"Je ne m'attendais pas forcément à une réaction comme ça (...) je me dis que ce n'est pas complètement anormal [qu'elle soit perdue et qu'elle n'arrive pas à faire l'intégralité de son programme], ce n'est pas parce que je suis complètement nulle et pas à ma place en tant qu'enseignante, peut être c'est qu'il y a trop de choses à faire et que c'est difficile de tout faire. C'est super important qu'elle ait 10 ans d'expérience [la réactante] (...) elle dit que ceux qui arrivent à tout faire, ce n'est pas vrai, ce n'est pas possible, qu'il faut se fixer des priorités (...) et c'est ce que je finis par faire dans les semaines qui ont suivi ». consistent à faire comprendre cela à l'initiatrice, et à l'aider à distinguer les contenus les plus importants (priorisation des contenus pédagogiques). On voit dans cet exemple que la participante se construit une représentation de l'expertise de son interlocuteur, dans laquelle l'expérience tient une place d'autorité. Les échanges entrainent un changement de point de vue et une diminution du sentiment d'incompétence ressentie par l'initiatrice «les contributions me rassurent, je me sens déjà mieux (...) c'est pas que ça me fait oublier les préoccupations concrètes, mais je me dis « bon, on va faire au maximum " (entretien). Ce soutien psychologique, ici traduit par le partage d'expérience similaire et l'aide à la réévaluation de la situation que proposent les réactants, permet à l'initiatrice de reprendre confiance en elle et de s'engager dans de nouvelles pratiques (ici ne plus appliquer l'intégralité du programme, mais faire des choix didactiques et pédagogiques).

\section{2. Élaboration collective d'outils et projets pédagogiques, souvent innovants}

61 pairs et demander des retours d'expériences, des avis ou des conseils. S'en suivent des échanges qui participent à une co-élaboration de méthodes pédagogiques où chacun contribue à la conception du projet ou de l'outil. Celui-ci peut être adapté à la situation de chacun ou peut être élaboré pour être mis en œuvre collectivement. Un exemple de projet collaboratif est celui de la création d'un "rallye cartes postales " qui consiste en l'envoi par une classe de chaque région de France et d'outre-mer d'une carte postale représentant sa région aux autres classes. L'objectif est de faire travailler les élèves sur la géographie des régions françaises, de manière ludique et participative. Cette activité est mise en place avec succès pour la $3^{\text {ème }}$ année consécutive et fédère de plus en plus de participants (onze « vagues » de rallyes cartes postales ont en 2015 lieu en parallèle).

Un autre exemple de projet, intitulé "la météo des écoles », permet de travailler de manière collaborative et interdisciplinaire. Ce projet a pour principal objectif de favoriser l'utilisation des Technologies de l'Information et de la Communication de façon transversale sur un projet à dominantes sciences et géographie, à travers un travail en réseaux d'écoles. Une page Internet a été créée sur laquelle une carte du monde apparait, les élèves des écoles participantes (adhésion libre et gratuite au projet) sont invitées à faire un relevé météorologique chaque jour sur la plateforme. Le site permet la saisie de différentes mesures (état du ciel, température, pression, force du vent, direction du vent, précipitations mensuelles, type de nuages) et la sortie des résultats sous différentes formes (cartes, tableaux, graphiques). Ainsi se construit une base de données donnant lieu à des suivis sous forme de cartes interactives, graphiques, tableaux, etc. Ces données servent ensuite de base à des travaux en géographie, sciences et vie de la terre, éducation 
civique, etc. Ce projet permet également de démarrer une correspondance électronique entre des écoles éloignées et aux cultures parfois différentes.

Les participants peuvent aussi concevoir collectivement des outils ad hoc. C'est le cas d'une enseignante chevronnée qui lance une discussion sur le thème du livret pédagogique, injonction ministérielle d'évaluation des compétences des élèves, auquel elle s'oppose. Les échanges de cette discussion tourneront autour de l'amélioration et l'adaptation de ce livret dont les compétences au départ sont décontextualisées, afin de le rendre un peu plus conforme à la réalité de l'observation des progrès des enfants. Ce livret, amélioré par une quinzaine de participants au fil des échanges, sera réinvesti par chacun dans leur classe. L'initiatrice de la discussion, avec laquelle un entretien a été réalisé, a expliqué que la conception collective avait été fructueuse de son point de vue, tant au niveau du soulagement que cela lui a procuré de trouver une solution « acceptable », qu'au niveau de la réutilisation de ce livret dans sa pratique :

«La discussion m'a aidé très très concrètement parce que (...) j'ai construit mes évaluations. Au niveau hyper concret, ça m'a vachement aidé. Et ça m'a calmé aussi, car j'étais très énervée. C'est comme toujours, quand on a un truc sur le cœur, ça fait du bien de le dire sinon ça aigrit. C'est toujours bien de pouvoir parler quand on en a gros sur la patate, c'est toujours bien aussi de voir qu'il y a des gens qui ne tombent pas dans le système et qui essaient d'une manière ou d'une autre de prendre de la distance » (entretien).

On voit ici le lien tenu entre amélioration de la pratique professionnelle et amélioration du bien-être, grâce aux échanges et à l'élaboration collective d'un outil pédagogique.

Les participants expriment du plaisir à travailler ensemble et à trouver collectivement des solutions ou à concevoir des outils pour leur pratique professionnelle. Cela est d'autant plus précieux à leurs yeux qu'ils manquent d'échanges de ce type au sein de leur établissement, faute d'avoir des homologues avoir des niveaux de classe identiques :

« ça fait vraiment du bien de pouvoir échanger avec des collègues qui ont le même niveau que nous (...) on se comprend, on s'entraide, on construit ensemble (...) ça m'aide vachement ce truc [le forum], je me sens mieux ».

\section{3. Élaboration de savoirs et savoir-faire}

Les échanges permettent aux participants d'élaborer des savoirs et des savoir-faire sur différents aspects de leur métier, notamment en ce qui concerne la gestion relationnelle. Les discussions sur le forum concernent peu les relations entre collègues, non qu'ils soient inexistants, mais en raison de la crainte de levée de l'anonymat et des représailles (Prost et al., 2014). Les échanges sur les aspects relationnels concernent principalement les élèves (parfois les parents d'élèves), et s'apparentent à des problématiques de gestion de classe et de posture à adopter face aux élèves. Cet aspect représente une part importante de leur travail et est peu pris en compte dans la formation des enseignants actuellement proposée. Celle-ci met l'accent sur la maitrise des contenus didactiques ce qui ne leur permet plus de se confronter à des situations réelles de classe. Aussi, de nombreux jeunes professionnels se trouvent désarmés lorsqu'ils arrivent dans leur établissement, confrontés à une classe d'élèves parfois difficiles. C'est la situation dans laquelle a été une jeune enseignante qui relate son vécu en entretien. Jeune mère de deux enfants de retour de congés maternité, elle hérite de la classe difficile de sa collègue qui ne l'a pas prévenue ni aidée en amont à trouver des solutions pour éviter des débordements. Assez vite, elle est dépassée par le comportement turbulent voir agressif 
de certains élèves, et ne trouve pas de soutien auprès de son institution. Elle traverse alors une période très difficile, où elle n'arrive plus à gérer, ni le comportement de ses élèves, ni le contenu de ses cours. Elle explique en entretien avoir été «en dépression pendant plusieurs mois", vivre une véritable "descente aux enfers". Suite à un événement particulier où des élèves lui dérobent sa chaussure et la mettent dans les toilettes, elle décide de chercher de l'aide sur le forum. Elle ne parle pas du manque de soutien de ses collègues dans les échanges, car elle sait que l'une d'elles se rend également sur cet espace et craint d'être reconnue. Elle concentre son propos sur ses problèmes de gestion de classe, et cherche des conseils avisés de pairs plus expérimentés.

«Ma préoccupation c'était aussi ceux qui ont envie de travailler, comment je les fais avancer (...) parce qu'il n'y a pas que les enfants en difficultés, qui mettent le bazar. Enfin, ils peuvent être en difficultés et être gérables et que les autres puissent avancer. Je cherchais quand même des moyens de pression pour les calmer » (extrait de l'entretien)

67 Les professionnels ayant participé à cette discussion ont souligné le côté inadmissible de cette situation, et ont insisté sur la nécessité d'alerter les parents et de se faire épauler par les collègues afin de mettre en place des mesures collectives. L'initiatrice a essayé de mettre en place ces conseils, mais les effets n'ont pas été ceux attendus, les parents étant tout aussi dépassés qu'elle, et ses collègues manifestement peu disposés à lui prêter main forte. Elle a fini par écrire une lettre à l'inspection académique pour dénoncer la situation avec les élèves, sur les conseils de plusieurs participants.

On voit ici que les expériences et conseils prodigués par les pairs sur le forum ont été mis en pratique dans le réel par l'initiatrice, qu'une partie d'entre eux n'ont pas été suffisants pour régler la situation, mais ont permis à cette professionnelle d'envisager d'autres alternatives que celles qu'elle avait mises en place. Parallèlement, les échanges empreints d'empathie, compression et soutien émotionnel lui ont procuré un soutien psychologique nécessaire dans cette période très difficile émotionnellement pour elle. Elle explique, en entretien, que les avis sans équivoque sur la nature anormale et inacceptable de la situation des participants l'ont énormément rassurée, car elle commençait à douter d'elle-même et du bien-fondé de son ressenti «ça m'a vachement rassurée, ça m'a rassurée dans le fait que ma classe était vraiment difficile et que ce n'était pas normal, que je n'étais pas complétement folle » (entretien).

Les trois quarts des participants (questionnaire) et l'ensemble des personnes interviewées déclarent que les échanges sur le forum les aident à trouver des solutions à des situations qui, dans leur pratique quotidienne, leur posent problème ${ }^{6}$.

«Ça m'aide beaucoup. Par exemple pour des élèves un peu chahuteurs, je suis en train de tester une méthode trouvée sur le forum, et pour le moment ça fonctionne bien » (questionnaire)

70 Cet exemple met en évidence un effet des échanges sur l'amélioration du bien-être des participants.

71 Les échanges permettent également le changement de point de vue, l'élargissement des idées, des possibilités d'action, ce qui in fine permet de rassurer la participante.

«Ça m'ouvre à d'autres points de vue, ça me donne des idées que je n'aurais pas eues, soit pour des nouvelles notions, ou pour l'autonomie, c'est surtout ça que je cherche en ce moment pour les CP et les CE1. On nous demande d'évaluer la production de l'écrit alors que les $\mathrm{CP}$ ne savent pas écrire. Du coup, je suis allée sur le forum et j'ai trouvé plein de choses là-dessus (...) ça m'aide vachement d'avoir plusieurs solutions possibles, ça me rassure » (entretien) 


\subsection{Les facteurs de conversion du forum} facilitent la capacité des membres individu à faire usage des ressources (forum) pour les " convertir » en réalisations concrètes. La première section s'attachera à présenter l'anonymat de l'identité réelle comme élément qui conditionne l'engagement des participants sur le forum ; la seconde sera centrée sur les éléments qui participent à faire $\mathrm{du}$ forum un espace réflexif sur ses propres pratiques à savoir une communauté de pairs réactive, orientée vers l'entraide, qui partage les mêmes pratiques professionnelles.

\subsubsection{Anonymat de l'identité réelle}

L'anonymat de l'identité réelle est une caractéristique propre aux échanges sur le forum très important dans l'engagement des professionnels. En effet, $70 \%$ des participants mettent en avant, via le questionnaire et en entretiens, que le principal avantage des échanges sur les forums, en comparaison des échanges plus conventionnels, est la situation d'anonymat. L'anonymat semble faciliter le dévoilement de soi :

"On est libre de dire ce que l'on ressent, ce qui peut être plus difficile dans un

établissement avec ses collègues ». ce qui leur permet de se livrer sur leurs pratiques et difficultés :

"C'est moins gênant de poser des questions bébêtes sur le forum parce que justement il y a ce côté anonyme donc on ose plus facilement. » (questionnaire).

L'anonymat est une préoccupation systématiquement évoquée par les participants en entretien. Ceux-ci expliquent par quelles stratégies ils passent pour le préserver et pour éviter des répercussions éventuelles. Dès lors, ils se montrent très attentifs à ne pas divulguer d'informations permettant de lever cet anonymat. Ils censurent ainsi des informations personnelles, mais aussi celles sur leurs collègues et élèves (pour plus de détails, voir Prost 2012 ; Prost et al., 2013). En témoignent les propos de cette participante qui s'est créé un second pseudonyme pour ne pas être reconnue et ainsi brouiller les pistes:

«J'avais vraiment tendance sur mon ancien profil à dire qui j'étais, où j'étais en poste et puis j'avais dans mes contacts mes copines, ma cousine alors cet été j'avais décidé de me créer un nouveau profil. (...) je ne voulais pas que des gens extérieurs puissent voir que j'étais là et se disent "qu'est-ce qu'elle fait là ?" " (entretien).

Le forum EDP étant très fréquenté par les professeurs des écoles, il est arrivé que des personnes reconnaissent leurs collègues au travers des informations données dans les messages. Une participante explique en entretien que sa collègue lui a fait une remarque suite à un message qu'elle avait posté sur le forum, ce qui (selon ses termes) l'a " gênée ", elle a pris soin par la suite de masquer des informations qui permettraient à d'autres de la reconnaître. Les informations sur les collègues ou la hiérarchie sont de ce fait très fréquemment évitées par peur d'être reconnu et des représailles par la hiérarchie, mais aussi par les parents.

«Il y plein de choses que je ne dis pas, car tu ne sais jamais où ça peut aller, surtout des choses sur la direction (...) si ça se passe mal avec un collègue, je dirai que je suis 
mal avec un de mes collègues, comme ça, il ne saura pas lequel. Je ferai attention. $\mathrm{Ou}$ alors ce que je ferai, mais je ne l'ai jamais fait, c'est créer un autre pseudo. Si vraiment j'ai un gros souci, je me crée un autre pseudo, il faut faire gaffe à ça vraiment. » (entretien).

«L'année dernière, j'avais un souci avec un élève, j'ai posté un message (...) je mettais des choses qui pouvaient permettre à quelqu'un de reconnaitre la personne dont je parlais (...) j'avais écrit "message volatil" c'est ce qu'on met quand on veut effacer la discussion et j'avais eu une réponse très rapide et la discussion s'était poursuivie en messages privés. » (entretien).

\subsubsection{Une communauté de pairs réactive qui partage les mêmes pratiques} qui participe à faire du forum un espace réflexif sur propres pratiques. Cette communauté est composée de nombreux membres qui s'engagent sur le forum dans une visée d'entraide et d'amélioration de leur pratique professionnelle. La recherche d'échanges avec un nombre important de pairs permet aux participants de s'assurer de trouver des pairs vivant (ou ayant vécu) des expériences similaires (70\% des répondants au questionnaire), autrement dit qui partagent un même référentiel.

dû, selon les participants à l'étude, à une faible cohésion d'équipe, à des conflits interpersonnels, à un manque de dialogue et d'entraide, et surtout à une absence de collègues partageant les mêmes pratiques professionnelles. Notons que ces professionnels n'ont pas systématiquement des problèmes relationnels avec leurs collègues, la plupart déclarent avoir de bonnes relations. Les collègues « directs " au sein des établissements sont d'ailleurs mentionnés par $90 \%$ des participants (questionnaire) comme des cibles du partage social ( $75 \%$ pour les collègues « indirects » du forum). Ceci tend à montrer que les participants au forum ne sont pas nécessairement des personnes isolées sur le lieu de leur travail, qu'ils échangent avec leurs collègues, mais ces échanges ne sont pas pleinement satisfaisants pour eux parce que cela ne les aide pas à résoudre les difficultés qu'ils rencontrent au niveau de leur pratique professionnelle. Ainsi, l'isolement n'est pas vécu ici comme la résultante d'un manque de relations socio-affectives mais celle d'une carence d'échanges épistémiques, sur le contenu même du travail :

«On est trois classes, une de maternelle, il y a une enseignante en CP-CE1 et du coup moi je suis toute seule pour les grands et c'est des contenus bien différents et des gestions de classe qui n'ont rien à voir (...) je suis un peu isolée» (témoignage lors d'un entretien).

Les participants s'engagent dans les forums parce qu'ils n'ont pas autour d'eux de possibilité de trouver des réponses aux questions qu'ils se posent. La question du partage d'un référentiel commun apparait donc centrale. Certains participants se sentent également démunis face au manque d'aide de leur hiérarchie (notamment les inspecteurs) dont ils ne perçoivent que la fonction évaluative de leur travail.

La richesse des réponses et la réactivité des membres constituent deux autres caractéristiques importantes pour l'engagement des membres. Le forum regroupe une quantité importante de professionnels avec des niveaux de classe et des pratiques pédagogiques différentes, ce qui garantit aux participants de trouver des homologues aux pratiques semblables avec qui discuter de leur métier. Le forum EDP est très fréquenté, ce qui accroit l'assurance d'obtenir rapidement des réponses. 
« Pendant vraiment un bon moment, on se retrouvait au quotidien, on était $7 \mathrm{ou}$ 8 instits et c'était vraiment très vivant (...) Et là, tous les jours en sortant du boulot, je me précipitais dessus, c'était vraiment mon suivi au quotidien et à la fin de l'année quand ça ne marchait plus trop parce que c'est bientôt les vacances, ça m'a manqué presque, je me disais "mince, où elles en sont", c'était des collègues, j'étais beaucoup plus au courant de ce qui se passait dans leur classe que ma collègue à l'école. » (entretien)

La mise à distance et la réflexivité sont facilitées par le caractère permanent des messages sur le forum, qui provoque chez les participants un sentiment de liberté. Une participante au forum interviewée explique la place importante de ce contrôle dans son engagement sur le forum qui traduit un sentiment de liberté :

«Ce qui est bien avec le forum, c'est que je peux contrôler ce que j'ai envie d'entendre ou si je veux parler. Si j'ai envie d'entendre parler du travail, j'y vais et si je ne veux pas, je le mets à l'écart. La dernière fois, on est allé manger chez ma copine et elle a parlé toute la soirée du boulot et j'en avais marre, mais je ne pouvais pas le dire."

Une autre caractéristique de la communauté de pairs est l'émergence de rôles en son sein. Les membres se créent une identité virtuelle et se construisent une représentation de leurs interlocuteurs au travers de leurs interventions. Dans notre étude, les participants expliquent clairement (en entretien) identifier plusieurs types de rôles, les "sauveurs ", les "enquiquineurs", les "spécialistes de la polémique», ceux qui "s'accaparent une discussion », etc. Tout un panel de rôles se dessine, au sein duquel s'en détache un, particulièrement reconnu par les enseignants en attente d'aide, qui correspond à «l'expert» du domaine. Ce rôle s'appuie sur l'expérience professionnelle des participants, les plus anciens dans le métier étant souvent considérés comme des experts lorsque leur témoignage est assorti d'une analyse. La construction de telles représentations est nécessaire en ce que cela leur permet d'identifier les compétences des autres membres, de reconnaître l'expertise et ainsi d'évaluer la validité du témoignage apporté. Ainsi, deux messages n'ont pas le même impact selon la personne qui en est l'émetteur.

«Avant de lire les messages, pour certains je connais le contenu. C'est vrai qu'il y a des rôles, il y a les sauveurs, les enquiquineurs (...). En général ce sont les vieux de la vieille qui sont très très actifs, il y a aussi quelques jeunes qui ont des blogs avec des mines de ressources et qui participent beaucoup sur le forum. Il y a 2 ou 3 anciens, notamment dans les directeurs, quand on pose une question, on peut être sûrs que dans l'heure qui suit on a des réponses. » (entretien)

« Il y a un sentiment d'appartenance, c'est clair (...) Quand je vois les pseudonymes des personnes, je n'ai aucune idée de leur visage, mais j'ai l'impression de les connaître. » (entretien)

«Il y en a (...), en voyant le pseudo d'une personne, je sais où elle a écrit, je connais à peu près son avis sur la question. Il y en a une que j'admire, j'adore leurs méthodes, je me dis « le jour où je serai comme elle... ( (entretien)

Bien que l'anonymat soit mentionné comme une condition au dévoilement de soi sur le forum, il est fréquent que les participants lèvent leur anonymat avec certains membres du forum en dehors de ce dernier. Environ la moitié des professionnels ayant répondu au questionnaire déclarent choisir révéler leur identité à certains membres, la rencontre physique ayant lieu dans un quart des cas. Cette levée d'anonymat peut être considérée comme un indicateur de la construction de collectif. C'est par exemple le cas d'un groupe de participantes chevronnées présentes sur le forum par militantisme, pour aider les plus jeunes à ne pas «faire de dégâts sur les gamins ». Ces personnes sont reconnues par les 
membres comme expertes et les entretiens montrent que leurs interventions ont un poids beaucoup plus important que les interventions d'autres membres. Ce petit groupe (de cinq ou six participantes) attend des échanges des réflexions méta sur le sens de leur métier, elles ont besoin d'un positionnement plus analytique de leur pratique, qu'elles peinent à trouver sur le forum. En effet, deux d'entre elles expriment en entretien que les échanges sont souvent pris par l'urgence du quotidien, par des problèmes pragmatiques qui nécessitent une intervention rapide. Aussi, pour pallier ce manque d'échanges sur ce représente le travail d'enseignants du primaire, ce que sont les missions de ce métier au regard de la société, elles ont décidé d'échanger entre elles par d'autres biais. On voit ici que le forum ne répond pas entièrement aux attentes de ces participantes, mais il a été un outil qui leur a permis de se connaître, de reconnaître leurs attentes et de les fédérer. Les attentes liées à des échanges plus analytiques et réflexifs sur la pratique ne sont ainsi pas assouvies sur EDP, et se produisent sur une autre scène, hors du forum. L'anonymat est alors levé, des liens nouveaux se créent entre ces personnes. Les échanges sur le forum permettent en effet aux participants de nouer des relations consistantes qui se prolongent, dans près d'une fois sur deux, par des modes de communication où l'anonymat est abandonné (téléphone, mail, courrier ou rencontre).

\section{Discussion}

La discussion des résultats sera organisée en deux points. Nous défendrons, dans le premier point, le fait que les échanges entre pairs sur le forum étudié sont source de développement de la pratique et du bien-être des professionnels, et reviendrons sur les facteurs d'engagement (ou de conversion) des participants sur le forum identifiés dans les résultats. Dans un second point, nous nous demanderons à quelles conditions un tel développement de la pratique et du bien-être pourrait être mis en place au travers d'une intervention au sein même des établissements.

\subsection{Le pouvoir d'agir comme développement de la pratique et du bien-être}

Dans notre étude, nous avons envisagé le pouvoir d'agir a été appréhendé au travers du développement des pratiques professionnelles (dimension constructive ou développementale - Falzon, 2013) et du développement du bien-être des individus (dimension émotionnelle). Le premier type de développement se traduit du point de vue pragmatique à travers l'acquisition de connaissances nouvelles sur les pratiques des pairs, et par la remobilisation en situation de travail des savoirs et savoir-faire ainsi échangés.

Les échanges qui se produisent sur le forum étudié ont des points de proximité avec ceux identifiés dans les groupes où se produit de l'activité réflexive (Mollo \& Nascimento, 2013), activité nécessaire à la construction compétences (Weill-Fessina \& Pastré, 2004). On trouve dans nos données les caractéristiques mises en évidence par ces auteures, à savoir que les échanges permettent aux professionnels, par la confrontation aux autres, de changer de point de vue (Clot, 2008, parle de renouvellement de sens donné aux situations); de prendre conscience des autres formes de réalisation de l'activité ; de faire une analyse critique de leurs propres savoirs et savoir-faire; et de construire de nouveaux savoirs ; caractéristiques auxquelles nous avons ajouté le réinvestissement des savoirs et savoir-faire dans l'activité professionnelle future. 
88 Nos résultats ont montré que ces pratiques réflexives, préalables au développement (Weill-Fessina \& Pastré, 2004) permettent l'élaboration de savoirs, de savoir-faire, de solutions et de normes notamment par rapport aux injonctions ministérielles parfois difficiles à mettre en œuvre; d'outils collectifs et de projets pédagogiques innovants (e.g. pratiques collectives et collaboratives d'enseignement). L'ensemble de ces activités produites sur cet espace virtuel fait écho à celles observées dans les communautés de pratique virtuelles (Murillo, 2008 ; Wenger, 1998).

89 Les professionnels peuvent discuter des directives ministérielles et des ajustements de leurs pratiques au regard de leurs contraintes, ce qui rappelle les travaux de Leplat (1997) sur la régulation de l'activité de travail, et l'instauration de marges de manœuvre situationnelles (Coutarel et al., 2015) dans le cadre de discussions collectives (Caroly, 2011 ; Mollo \& Nascimento, 2013). Les participants construisent des connaissances sur la manière de faire face à un problème (Weill-Fessina \& Pastré, 2004), au travers de la mise en récit de leurs expériences considérée comme une aide à la capitalisation des connaissances et à la construction des pratiques professionnelles (Beaujouan et al., 2013 ; Bourassa et al., 1999 ; Soulier, 2006).

90 Le développement du bien-être, et plus largement de la santé, n'est pas à distinguer de ce mouvement épistémique. Nos résultats ont montré que la dimension émotionnelle, traduite par le témoignage de soutien émotionnel et l'amélioration de l'expérience émotionnelle, est omniprésente dans les fils de discussion. Le partage social des émotions, qui a lieu habituellement avec la sphère privée (amis, famille) (Rimé, 2005) semble ici se produire entre pairs qui ne se connaissent a priori pas. Les participants expriment massivement un besoin de décharge émotionnelle, de partage d'émotions et de soutien psychologique pour faire face aux situations qu'ils traversent. Le témoignage de vécu similaire, la réassurance et la compréhension des situations sont des manifestations de soutien fréquemment retrouvées dans les échanges, à l'instar des communautés de soutien en ligne (e.g. Derks et al., 2007 ; Malik \& Coulson, 2008 ; Pfeil \& Zaphiris, 2007). Avoir vécu une situation analogue et faire part de son ressenti associé ainsi que des solutions trouvées pour résoudre le problème semble être une forme de soutien tout à fait attendu et apprécié par les participants. Cela atteste, en creux, de la compréhension de la situation par l'interlocuteur dont les propos acquièrent plus de crédibilité aux yeux de l'initiateur (Walther \& Boyd, 2002). Cela peut expliquer, dans nos résultats, que les professionnels plus expérimentés ont une « autorité » plus importante que les novices.

91 Outre le témoignage de soutien émotionnel observé, les échanges permettent souvent une amélioration de l'expérience émotionnelle des participants, ce qui va dans le sens de certaines études qui postulent que les échanges au sein des communautés de soutien en ligne ont un effet bénéfique sur le bien-être des participants (e.g. Braithwaite et al., 1999 ; Malik \& coulson, 2008 ; Pfeil et al., 2009 ; Wright \& Bell, 2003). L'amélioration de l'état émotionnel peut être le résultat d'une résolution de problème; autrement dit, avoir trouvé une solution à la difficulté engendre une amélioration de l'état émotionnel. Les échanges permettent un apprentissage à mieux réguler les situations à venir et réduisent ainsi le vécu émotionnel négatif des professionnels qui se sentent mieux " armés " pour affronter les situations à venir. Il s'agit de "réassurance socio-cognitive » (Rimé, 2005). L'augmentation de la capacité d'agir (possibilités d'action) permet une diminution de la souffrance psychique (Clot, 2004), et diminue les risques psycho-sociaux grâce au collectif (Marc et al., 2011). L'amélioration de l'état émotionnel peut aussi être le préalable à la résolution de difficultés ou à un changement de pratique (je me sens plus serein grâce au 
constat que d'autres vivent une situation similaire ce qui me permet d'envisager plus tranquillement des solutions pour résoudre mon problème ou ma difficulté).

Hormis les travaux de la clinique de l'activité qui mettent en avant l'importance des affects pour le développement de l'activité, l'aspect émotionnel est généralement passé sous silence lorsqu'il est question du développement du pouvoir d'agir. Pourtant, le versant émotionnel semble capital, le développement que nous proposons d'appeler " pouvoir d'agir sereinement » est implicitement attendu dans une organisation qui non seulement permet d'élargir le rayon d'action des sujets (Clot \& Simonet, 2015), mais qui le fait sans ajouter une pression qui rendrait ce pouvoir émotionnellement éprouvant.

L'étude a permis de mettre en évidence des facteurs qui permettent la conversion des capacités d'agir en pouvoir d'agir (Fernagu-Oudet, 2012, 2016). La ressource à disposition (i.e. le forum) permet le passage de potentialités en réalisations concrètes (i.e. développement de la pratique et du bien-être des participants)

L'anonymat permet aux professionnels de se livrer plus librement sur leur vécu émotionnel, comme c'est le cas dans les communautés de soutien (e.g. Pfeil \& Zaphiris, 2007 ; Preece, 1999), mais aussi sur ce qu'ils ne savent pas faire, ne comprennent pas, ne font pas correctement, voudraient faire différemment. Le second facteur de conversion renvoie à la présence d'une communauté de pairs réactive, bienveillante et ayant un référentiel commun. L'émergence de rôles, comme c'est le cas dans des communautés comme celles de conception de logiciels open source ou de conception d'encyclopédie comme Wikipedia (Barcellini, Détienne, \& Burkhardt, 2014 ; Détienne, Baker, Fréard, Barcellini, Denis, \& Quignard, 2016), est une trace de la structuration de la communauté. De nouveaux collectifs se forment au sein de ces espaces, les participants se reconnaissent (pseudonyme), ce qui leur permet de maintenir dans le temps des échanges, voire de passer à des échanges dans le « réel » (comme c'est le cas dans d'autres communautés en ligne, e.g. Parks \& Floyd, 1996).

95 L'anonymat, le partage de pratiques et l'accès permanent assurent à chacun la liberté de choix, primordiale dans la théorie des capabilités (Fernagu-Oudet, 2012, 2016; Sen, 2001, 2003) : choix de la fréquence et des moments de consultation, choix de ce qui est dévoilé aux autres et choix de confronter ses pratiques pour les faire évoluer. Les participants ont la possibilité de seulement lire les contributions (activité de lurking) et s'en aller aussitôt si la discussion n'entre pas en résonance avec leurs préoccupations. Le lurking représenterait $90 \%$ de l'activité des forums (Nonnecke \& Preece, 2000).

Si le lien entre pouvoir d'agir et développements de la pratique et du bien-être a été fait dans cette étude, il convient d'en mentionner les limites. L'étude est limitée à un unique forum de discussion conçu pour les enseignants du primaire. Ce métier, très féminisé, a des caractéristiques qui lui sont propres et qui ne se retrouveraient probablement pas dans d'autres. Parmi ces particularités, nous pouvons notamment citer que les professionnels ont un manque de formation, qu'ils ont de larges marges de manœuvre larges, mais ne savent pas toujours quoi choisir, et n'ont pas assez d'échanges sur leur pratique professionnelle au sein de leurs établissements. Ces caractéristiques ont peutêtre un poids important pour que les forums soient source de développement.

Également, nous aurions souhaité interviewer davantage de personnes, car c'est une possibilité de creuser, à partir du vécu des situations la question du développement de la pratique professionnelle et du bien-être. Cependant, obtenir des entretiens s'avère 
difficile, certainement dû au fait que les participants vont sur le forum pour rester anonymes et ne souhaitent pas mettre à jour leurs difficultés.

Enfin, le pouvoir d'agir est envisagé dans notre étude dans la mise en récit de l'activité, qu'elle soit observée dans les échanges virtuels ou dans les entretiens et questionnaires. Une analyse in situ sur le lieu d'exercice du métier pourrait venir compléter nos résultats.

\subsection{Un tel développement est-il envisageable au sein même des établissements?}

L'acceptation de la technologie - ici le forum - (Bobillier Chaumon, 2016) par les participants semble tenir au fait que celle-ci a été conçue en dehors des institutions, par et pour les professionnels eux-mêmes, et en lien avec les facteurs de conversion identifiés (anonymat et communauté de pairs bienveillante partageant les mêmes pratiques). Le développement de la pratique et du bien-être semble être soumis à ces conditions, préalables à l'engagement des professionnels. Les échanges sur le forum permettent de nouvelles possibilités de développement aux professionnels, qui sans celui-ci, ne pourraient voir le jour faute d'opportunités au sein des établissements. Les enseignants du primaire ont peu l'occasion d'échanger, au sein de leur institution, avec des pairs ayant les mêmes niveaux et étant confrontés aux mêmes problématiques. Les relations entre collègues peuvent être bonnes, mais il semble souvent manquer un soutien instrumental au niveau de la pratique même du métier, ce qui peut engendrer au fil du temps un sentiment d'isolement (Marc et al., 2011). Les échanges viennent combler les lacunes organisationnelles, notamment en ce qui concerne la formation des futurs enseignants qui ne leur donne pas suffisamment d'outils pour affronter la réalité du travail. Ainsi, ils pallient ce manque en investissant le forum au sein duquel ils se tournent vers des enseignants plus expérimentés qui leur fourniront les aides. Aussi, de tels groupes d'échanges sont-ils possibles dans les institutions au travers d'une intervention de type ergonomique?

100 Selon la clinique de l'activité, le développement se produit à travers l'intervention d'une tierce personne dans l'activité des sujets, faisant naître des controverses professionnelles sources de développement (Clot \& Simonet, 2015). Cette approche défend l'idée d'un nécessaire décontenancement affectif provoqué par le chercheur ou le collègue (Bonnemain, 2015). Or ici, les professionnels n'ont pas besoin d'être décontenancés affectivement puisqu'ils le sont déjà a priori et que c'est ce « décontenancement » qui justifie leur présence sur le forum.

101 Dans l'approche de l'ergonomie constructive (e.g. Arnoud \& Falzon, 2013; Mollo \& Nascimento, 2013), l'ergonome met en place des groupes de discussion ou lieux d'échanges que les professionnels peuvent s'approprier, et mettre en place par euxmêmes par la suite; des environnements qui donnent aux individus les moyens de développer leur autonomie de choix à agir de telle ou telle manière (FernaguOudet, 2016), leurs marges de manœuvre situationnelles (Coutarel et al., 2015). Dans notre étude, les échanges sont mis en place sans l'intermédiaire d'un tiers extérieur. Les participants viennent de façon totalement autonome participer à ces groupes de discussion, certes modulés par un modérateur qui garantit le respect des règles du forum (propos non injurieux, etc.), mais au sein desquels ils ont toute liberté de dévoiler ou non des informations sur eux-mêmes. 
Dans ce contexte, la mise en place de groupe d'échanges au sein même des institutions semble soumise à un certain nombre de difficultés qu'il conviendrait de dépasser pour en garantir le succès. Cela nécessiterait de recréer ces opportunités identifiées au travers des facteurs de conversion précédemment mentionnés. Notamment, le dispositif devrait mettre les enseignants suffisamment en confiance pour qu'il procède au dévoilement de soi qui a lieu sur le forum sous condition d'anonymat de l'identité réelle (ce qui peut paraître compliqué au vu de l'individualisme que rapportent de nombreux enseignants sur leur corps professionnel). Faire part de ses difficultés (e.g. relationnelles avec les collègues) et mettre à jour son sentiment d'incompétence demanderait des ajustements importants pour que cela ait lieu dans des espaces institutionnalisés. De même, le sentiment de liberté que les participants semblent apprécier en ce qu'ils peuvent aller quand ils le souhaitent sur le forum, apparait difficilement envisageable dans la vie « réelle » où les règles sociales les contraignent à participer aux discussions dont ils n'ont pas nécessairement besoin ou envie. Pour autant, nombre de travaux cherchent à mettre en place de tels collectifs pour que les individus se développent au sein même de leurs organisations (e.g. Arnoud \& Falzon, 2014 ; Rocha, 2014).

Dans le forum étudié, les difficultés se discutent, s'échangent et amènent à des changements de points de vue et développements des capacités «en dehors» de l'institution scolaire; elles sont donc invisibles pour l'organisation et ne permettent pas que celle-ci se transforme. Des compétences se construisent, des perspectives sur l'activité se confrontent, mais ce «en dehors des lieux du travail ». Les professionnels se développent, mais nous pouvons nous demander si les collectifs de travail et l'organisation sont également transformés, autrement que de façon indirecte et marginale. Dans notre étude, les sources de tension identifiées sont les rapports aux collègues, à la hiérarchie de l'établissement, à l'Éducation Nationale au travers de son inspection et de ses directives difficiles à comprendre et à mettre en place. Les facteurs de risques psychosociaux comme le sentiment d'isolement professionnel (Marc et al., 2011) sont un levier pour faire évoluer les pratiques organisationnelles. Ces échanges pourraient être une occasion pour l'institution de se rendre compte de ce qui dysfonctionne, de mettre en visibilité du travail empêché et de l'impossibilité de faire ln beau travail (Clot, 2008). En particulier, les forums pourraient être une aide précieuse aux concepteurs de la formation des enseignants pour identifier ses insuffisances. Une future recherche pourrait s'intéresser aux conditions à mettre en place pour que ce développement puisse se produire au sein même de l'institution, permettant ainsi le développement des individus, des collectifs et des organisations (Barcellini, 2015 ; Mollo \& Nascimento, 2013).

\section{BIBLIOGRAPHIE}

Arnoud, J., \& Falzon, P. (2013). La co-analyse constructive des pratiques. In P. Falzon (Ed.).

Ergonomie constructive (pp. 223-236). Paris : Presses Universitaires de France.

Bardin, L. (1977). L'analyse de contenu. Paris : PUF. 
Barcellini, F. (2015). Développer des interventions capacitantes en conduite du changement. Comprendre le travail collectif de conception, agir sur la conception collective du travail. Thèse d'habilitation à diriger les recherches, Université de Bordeaux.

Barcellini, F., Détienne, F., \& Burkhardt, J.M. (2014) A situated approach of roles and participation in Open Source Software Communities. Human-Computer Interaction, 4(3), 205-255.

Beaujouan, J., Coutarel, F., \& Daniellou, F. (2013). L'expérience des autres dans la formation : apports et limites du récit professionnel. Éducation permanente, 196(3), pp25-38.

Bobillier Chaumon, M.E. (2016). Acceptation située des TIC dans et par l'activité : premiers étayages pour une clinique de l'usage. In M.E. Bobillier Chaumon \& M. Dubois (Eds.). Numéro spécial Psychologie du Travail et des Organisations.

Bonnemain, A. (2015). Les paradoxes de l'intensité affective dan sl'autoconfrontation. L'exemple de l'activité dialogique des chefs d'équipe de la propreté de Paris. Thèse de doctorat, CNAM Paris.

Bonvin JM., \& Farvaque N., (2007), L'accès à l'emploi au prisme des capabilités, enjeux théoriques et méthodologiques, Formation emploi, 98

Bourassa, B., Serre, F., \& Ross, D. (1999). Apprendre de son expérience. Presses Universitaires du Québec.

Braithwaite, D. O., Waldron, V. R., \& Finn, J. (1999). Communication of social support in computer-mediated groups for people with disabilities. Health Communication, 11(2), 123-151.

Cahour, B. (2006). Les affects en situation d'interaction coopérative : proposition méthodologique. Le Travail Humain, 69(4), 379-400.

Cahour, B., Salembier, P., \& Zouinar, M. (à paraitre). Analysing the lived experience of the activity. Le Travail Humain.

Caplan, E.C. (2003). Preference for Online Social Interaction. A theory of Problematic Internet Use and Psychosocial Well-Being, Communication Research, 30(6), 625-648.

Caplan, S., \& Turner, J. (2007) Bringing theory to research on comuter-mediated conforting communication. Computers in human behavior, 23(2), 985-998.

Caroly, S. (2011). Activité collective et réélaboration des règles comme ressources pour la santé psychique : le cas de la police nationale. Le Travail Humain, 74(4), 365-389.

Caroly, S., \& Barcellini, F. (2013). Le développement de l'activité collective. In P. Falzon (Ed.), Ergonomie constructive (pp. 33-46). Paris : Presses Universitaires de France.

Charlier, B., \& Henri, F. (2004). Démarche d'évaluation, communauté de pratique et formation professionnelle. Revue suisse des sciences de l'éducation, 26(2).

Clot, Y. (2004). Travail et sens du travail. In P. Falzon (Ed.), Traité d'ergonomie (pp. 317-331). Paris : PUF.

Clot, Y. (2008). Travail et pouvoir d'agir. Paris : PUF.

Clot, Y. \& Simonet, P. (2015). Pouvoirs d'agir et marges de manœuvre. Le Travail Humain, 78(1), $31-52$.

Coutarel, F., Caroly, S., Vézina, N., \& Daniellou, F. (2015). Marges de manoeuvre situationnelle et pouvoir d'agir : des concepts à l'intervention ergonomique. Le Travail Humain, 78(1), 9-29.

Détienne, F., Baker, M., Fréard, D., Barcellini, F., Denis, A., \& Quignard, M. (2016). The Descent of Pluto: Interactive dynamics, specialisation and reciprocity of roles in a Wikipedia debate, International Journal of Human-Computer Studies, 86, 11-31. 
Derks, D., Fischer, A. H., \& Bos, A. E. R. (2007). The role of emotion in computer-mediated communication: A review. Computers in Human Behavior, 24(3), 766-785.

Engestrom, Y. (1987). Learning by expanding. Helsinki: Orienta-Konsultit.

Falzon, P. (2013). Ergonomie constructive. Paris : PUF.

Fernagu-Oudet, S. (2012). Favoriser un environnement « capacitant » dans les organisations. In E. Bourgeois \& M. Durand (Eds.), Former pour le travail (pp. 201-213). Paris : PUF.

Fernagu-Oudet S. (2014). Agir collectif et environnement capacitant, Éducation Permanente, Hors série AFPA.

Fernagu-Oudet, S. (2016). L'approche par les capabilités au prisme de la formation : vers la conception d'environnements capacitants, In S. Fernagu-Oudet \& C. Batal (Eds.), (R)évolution du management des ressources humaines. Des compétences aux capabilités. Presses Universitaires du Septentrion.

Gouedard, C., \& Rabardel, P. (2012). Pouvoir d'agir et capacité d'agir : une perspective méthodologique ? Illustration dans le champ de la santé, sécurité et conditions de travail. PISTES, 14(2).

Helgeson, V. S., \& Gottlieb, B. H. (2000). Support groups. In S. Cohen, L. G. Underwood, \& B. H. Gottlieb (Eds.), Social support measurement and intervention: A guide for health and social scientist (pp. 221-245). New York: Oxford University Press.

Hian, L. B., Chuan, S. L., \& Trevor, T. M. K. (2004). Getting to know you: exploring the development of relational intimacy in CMC. Journal of computer-mediated communication, $9(3)$.

Joinson, A. (2001). Self-disclosure in computer-mediated communication: The role of selfawareness and visual anonymity. European journal of social psychology, 31(2), 177-192.

Lave, J., \& Wenger, E. (1991). Situated learning: Legitimate peripheral participation. Cambridge University Press.

Leplat, J. (1997). Regards sur l'activité en situation de travail : contribution à la psychologie ergonomique. Paris : PUF.

Lewkowicz, M., Marcoccia, M., Atifi, H., Bénel, A., Gaglio, G., Gauducheau, N., \& Tixier, M. (2008). Online Social Support: Benefits of an Interdisciplinary Approach for Studying and Designing Cooperative Computer-Mediated Solutions. Proceedings of the 8th Conference on the Design of Cooperative Systems, pp. 99-110.

MaKinster, J. G., Barab, S. A., Harwood, W. S., \& Andersen, H. O. (2006). The effect of social context on the reflexive practice of pre-service science teachers: Incorporating a Web-supported community of teachers. Journal of Technology and Teacher Education, 14(3), 543-579.

Malik, S. H., \& Coulson, N. S. (2008). Computer-mediated infertility support groups: an exploratory study of online experiences. Patient Education and Counseling, 73(1), 105-113.

Maloney-Krichmar, D., \& Preece, J. (2005). A multilevel analysis of sociability, usability, and community dynamics in an online health community. ACM Transactions on Computer-Human Interaction (TOCHI), 12(2), 201-232.

Marc, J., Grosjean, V., \& Marsella, C. (2011). Dynamique cognitive et risques psychosociaux : isolement et sentiment d'isolement au travail. Le Travail Humain, 74(2), 107-130.

Merseth, K. K. (1992). First aid for first-year teachers. Phi Delta Kappan, 73(9), 678-683. 
Mollo, V., \& Nascimento, A. (2013). Pratiques réflexives et développement des individus, des collectifs et des organisations. In P. Falzon (Ed.). Ergonomie constructive (pp. 207-221). Paris: PUF.

Murillo, E. (2008). Searching Usenet for virtual communities of practice: using mixed methods to identify the constructs of Wenger's theory. Information Research, 13(4).

Nonnecke, B., \& Preece, J. (1999). Shedding light on Lurkers in Online Communities. Ethnographic Studies. Real and Virtual Environments: Inhabited Information Spaces and Connected Communities (pp. 123-128). Edinburgh: Ed. K. Buckner.

Parks, M.R., \& Floyd, K. (1996). Making friends in cyberspace. Journal of computer-mediated communication, 1(4), 80-97.

Pfeil, U., \& Zaphiris, P. (2007). Patterns of Empathy in Online Communication. Présenté à CHI 2007 - Emotion \& Empathy, San Jose, CA, USA.

Pfeil, U., Zaphiris, P., \& Wilson, S. (2009). Older adults' perceptions and experiences of online social support. Interacting with computers, 21, 159-172.

Preece, J. (1999). Empathic communities: Balancing emotional and factual communication. Interacting with computers, 12(1), 63-77.

Preece, J., Maloney-Krichmar, D., \& Abras, C. (2003). History of emergence of online communities. Encyclopedia of Community. Berkshire Publishing Group, Sage.

Prost, M. (2012). Les échanges entre professionnels de l'éducation : entre soutien psychologique et acquisition de connaissances sur la pratique. Thèse de doctorat, Télécom ParisTech, Université Pierre et Marie Curie.

Prost, M., Cahour, B., \& Détienne, F. (2013). Masquage des émotions et des attentes de soutien psychologique sur des forums d'entraide professionnelle. Activités, 10(2), 20-38. http:// activites.revues.org/699.

Prost, M., Cahour, B., \& Détienne, F. (2014). Le partage d'émotions et de connaissances sur la pratique : dynamiques des échanges dans les communautés de pratique virtuelles de professionnels. Le Travail Humain, 77(2), 177-202.

Prost, M., \& Fernagu-Oudet, S. (2016). L'apprenance au prisme de l'approche par les capabilités. Éducation permanente.

Rabardel, P. (1985). Les hommes et les technologies. Paris : Armand Colin

Rabardel, P. (2005). Instrument, activité et développement du pouvoir d'agir, Recherches, 251-265.

Rimé, B. (2005). Le partage social des émotions. Paris : PUF.

Rocha, R. (2014). Du silence organisationnel au développement du débat structuré sur le travail : les effets sur la sécurité et sur l'organisation. Thèse de doctorat, Université de Bordeaux.

Samurçay, R., \& Rabardel, P. (2004). Modèles pour l'analyse de l'activité et des compétences, propositions. In R. Samurçay \& P. Pastré (Eds.), Recherches en didactique professionnelle. Toulouse : Octarès.

Sen A., (2001), Development as freedom, Oxford: Oxford University Press.

Sen A., (2003), Un nouveau modèle économique. Développement, justice, liberté. Paris : Odile Jacob.

Soulier, E. (2006). L'acquisition de connaissances diférentielles dans l'interaction narrative : théorie de la descriptibilité pour la cognition distribuée. In E. Soulier (Eds.), Le storytelling, concept, outils et applications (pp. 117-176). Paris: Lavoisier. 
Tichon, J. G., \& Shapiro, M. (2003). The process of sharing social support in cyberspace. CyberPsychology \& Behavior, 6(2), 161-170.

Tidwell, L.C., \& Walther, J. (2002). Computer-mediated communication effects on disclosure impressions, and interpersonnal evaluations: getting to know one another a bit at a time. Human communication research, 28, 317-348.

Vermersch, P. (1994). L'entretien d'explicitation. Paris.

Vézina, M. (2002). Stress au travail et santé psychique : rappel des différentes approches. In M. Neboit \& M. Vézina (Eds.). Stress au travail et santé psychique (pp. 47-58). Toulouse : Octarès Vygotski L. (1934/1997) (3éd), Pensée et langage. Paris : La Dispute.

Walther, J., \& Boyd, S. (2002). Attraction to computer-mediated social support. In C.A. Lin \& D.J. Atkin (Eds.), Communication technology and society: audience adoption and uses (pp. 153-188). Hampton Press : Creekill.

Weill-Fassina, A., \& Pastré, P. (2004). Les compétences professionnelles et leur développement. In P. Falzon (Ed.). Ergonomie (pp. 213-231). Paris : PUF.

Wenger, E., (1998). Communities of Practice: Learning, Meaning and Identity. New York: Cambridge University Press.

Wills, T. A. (1981). Downward comparison principles in social psychology. Psychological Bulletin, 90 (2), 245.

Wright, K. (2002). Social support within an on-line cancer community: An assessment of emotional support, perceptions of advantages and disadvantages, and motives for using the community from a communication perspective. Journal of Applied Communication Research, 30(3), 195-209.

Wright, K. B., \& Bell, S. B. (2003). Health-related support groups on the Internet: Linking empirical findings to social support and computer-mediated communication theory. Journal of Health Psychology, 8(1), 39-54.

\section{NOTES}

1. Les marges de manœuvre situationnelles sont définies par les auteurs comme « la possibilité pour l'opérateur, dans une situation précise, d'élaborer un mode opératoire efficient (c'est-à-dire efficace pour la performance et compatible avec la préservation de soi, voire le développement de soi par le travail)» (Coutarel et al., 2015, p. 15).

2. «Le partage social implique deux éléments: la réévocation de l'émotion sous forme d'un langage socialement partagé, et la présence, au moins à un niveau symbolique, d'un partenaire auquel cette réévocation est adressée " (Rimé, 2005, p. 86)

3. Sur la période 2011-2015, 83 \% des professeurs des écoles sont des femmes d'après l'Institut des statistiques de l'Organisation des Nations Unies pour l'éducation, la science et la culture (UNESCO).

4. Source : http://www.education.gouv.fr

5. Le questionnaire n'a pas été joint en raison de la taille et des différents «chemins » de réponses. Pour plus de détails, voir Prost, 2012.

6. La définition de "problème » est différente pour chacun des participants. Nous l'entendrons ici dans une acception large, allant du simple questionnement à des situations plus complexes de résolution de problème, ou encore de dilemme éthique. 


\section{RÉSUMÉS}

L'étude se situe dans la perspective de l'ergonomie constructive (Falzon, 2013) et cherche à savoir si le forum d'enseignants du primaire étudié est une ressource qui permet le développement du pouvoir d'agir des participants, et à quelles conditions ceux-ci s'engagent sur le forum (facteurs de conversion - Fernagu-Oudet, 2012 ; Sen, 2001). Le pouvoir d'agir est envisagé sous l'angle du développement des pratiques (dimension constructive) et du développement du bien-être (dimension émotionnelle). Trois méthodes complémentaires de recueil de données ont été mises en place: (1) un questionnaire diffusé sur le forum EDP (quatre-vingts participants), (2) neuf entretiens d'auto-confrontation basés sur les traces écrites des discussions, et (3) vingt fils de discussions sélectionnés sur le forum. Une analyse de contenu a été réalisée sur l'ensemble des données à partir des catégories proposées par Mollo et Nascimento (2013) concernant les effets bénéfiques de l'activité réflexive, ainsi que des catégories liées à la dimension émotionnelle (témoignage de soutien émotionnel et amélioration de l'expérience émotionnelle). Les résultats mettent en évidence l'apport développemental des échanges au niveau de l'élaboration collective d'outils pédagogiques, de règles de métier, de savoirs et de savoir-faire. Le versant émotionnel est présent dans tous les échanges. Deux facteurs de conversion ont été identifiés : l'anonymat de l'identité réelle et la présence d'une communauté de pairs bienveillante, réactive et partageant les mêmes pratiques. Les résultats sont discutés au regard du développement de la pratique et du bien-être des professionnels, ainsi que des conditions nécessaires pour qu'un tel développement puisse avoir lieu au sein même des établissements.

The study explores whether the primary school teachers' forum for education professionals (EDP) is a resource that develops the empowerment of participants, and if so, under what conditions (conversion factors - Fernagu-Oudet, 2012 ; Sen, 2001). The power to act is examined from the standpoint of developing practices and well-being. We employed three complementary methods: (1) a questionnaire posted on the EDP forum (eighty participants), (2) nine selfconfrontation interviews based on written traces of these discussions, and (3) twenty discussion threads on the forum. A content analysis from the categories proposed by Mollo and Nascimento (2013) was performed (beneficial effects of reflexive activity, and emotional dimension). The results highlight the developmental contribution of exchanges, specifically for the collective development of educational tools, profession rules, and knowledge. The emotional aspect is present in all exchanges. The study also revealed two conversion factors : a) anonymity and b) the presence of a reactive and benevolent community of peers sharing the same practices. The results are discussed in relation to the development of the practices and well-being of professionals, and to the conditions for such a development taking place within schools.

\section{INDEX}

Mots-clés : pouvoir d'agir, échanges virtuels entre professionnels, développement des pratiques, développement du bien-être, facteurs de conversion

Keywords : power to act, virtual exchanges between professionals, development of practices, development of well-being, conversion factors Article soumis le, accepté le 


\section{AUTEURS}

MAGALI PROST

Université Paris Ouest Nanterre La Défense - magali.prost@u-paris10.fr

BÉATRICE CAHOUR

Télécom Paristech - beatrice.cahour@telecom-paristech.fr

\section{FRANÇOISE DÉTIENNE}

Télécom Paristech - francoise.detienne@telecom-paristech.fr 